\title{
Using real interpolation method for adaptive identification of nonlinear inverted pendulum system
}

\author{
Phu Tran Tin ${ }^{1}$, Tran Hoang Quang Minh ${ }^{2}$, Tran Thanh Trang ${ }^{3}$, Nguyen Quang Dung ${ }^{4}$ \\ ${ }^{1}$ Faculty of Electronics Technology, Industrial University of Ho Chi Minh City, Ho Chi Minh City, Vietnam \\ ${ }^{2}$ Optoelectronics Research Group, Faculty of Electrical and Electronics Engineering, \\ Ton Duc Thang University, Ho Chi Minh City, Vietnam \\ ${ }^{3}$ Faculty of Electrical and Electronics Engineering, Ho Chi Minh City University of Food Industry, \\ 140 Le Trong Tan, Ho Chi Minh City, Vietnam \\ ${ }^{4}$ Faculty of Electrical and Electronics Engineering, Ton Duc Thang University, Ho Chi Minh City, Vietnam
}

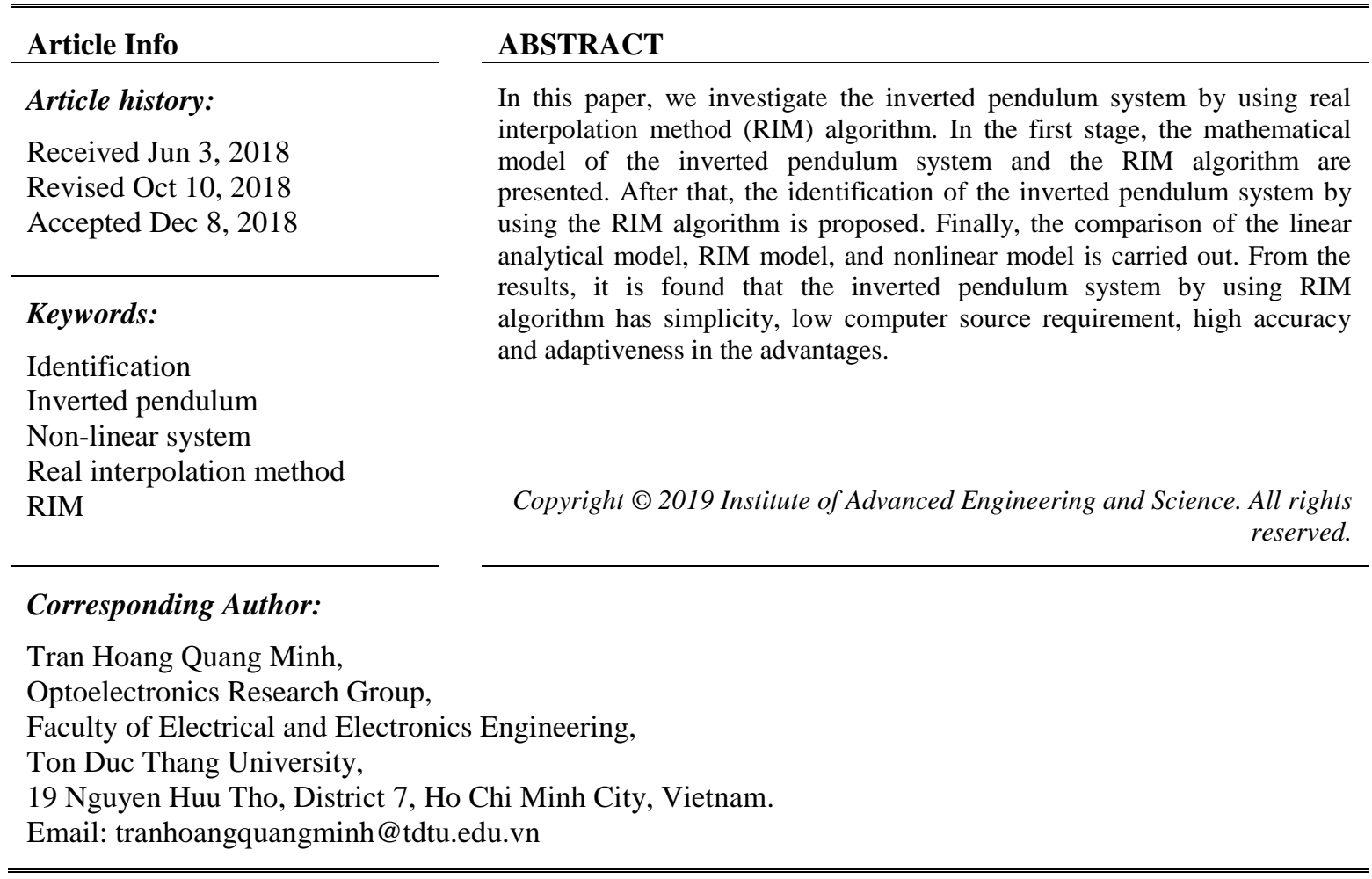

\section{INTRODUCTION}

Inverted pendulum system, which is an underactuated mechanical system and exhibits strong nonlinearity, has been considered as an exciting and challenging problem in automation. Notably, their features such as swing up, stabilization problem, higher order, nonlinear, robust coupling, and multivariate have been attracted more and more attention from control researchers for the last decades. Moreover, inverted pendulum system is widely used in the field of robotics and aerospace field, and so has essential significance both in the field of the theoretical study research and practice [1]-[3]. There are a lot of researchers about most popular types inverted pendulums such as the rotational single arm pendulum, the cart inverted pendulum, and the double inverted investigated the inverted pendulum by switching two different control laws [3], and in [4] LQR, double-PID and pole placement control techniques are used to control a cart inverted pendulum system. Moreover, fractional order PID controllers are proposed in [5] and [6] for control of the inverted pendulum system. In [7], a simulation study on PID and LQR control of a cart inverted pendulum system is carried out with and without disturbance input. Furthermore, in [8], control of a cart inverted pendulum on an inclined surface is performed using fuzzy and PID controllers. An ANFIS controller is proposed in [9] as an intelligent control technique to control an inverted pendulum. In addition, in [10], sliding mode controller is proposed to control a cart inverted pendulum system in both simulation and real- 
time implementation. From this point of view, research in the field of inverted pendulum system is still a hot direction in both theory and practice automation area.

In this paper, we investigate the inverted pendulum system by using real interpolation method (RIM) algorithm. In the first stage, the mathematical model of the inverted pendulum system and the real interpolation method (RIM) algorithm are presented. After that, the identification of the inverted pendulum system by using the real interpolation method (RIM) algorithm is proposed. Investigated and derived. Finally, the analytical algorithm derived by Simulink Mat Lab Software. From the results, it is found that the inverted pendulum system by using real interpolation method (RIM) algorithm is simplicity, low computer source requirement, high accuracy. The main contributions of this paper are presented as the following:

1) The mathematical model of the cart inverted pendulum system is proposed.

2) The identification of the inverted pendulum system by using the real interpolation method (RIM) algorithm is presented and derived.

The rest of the paper is proposed as follows. The cart inverted pendulum system, the real interpolation method (RIM) and the identification of the inverted pendulum system by using the real interpolation method (RIM) algorithm is introduced in Section 2. After that, some results and discussions are given in Section 3. Finally, conclusions are provided in Section 4.

\section{RESEARCH METHOD}

\subsection{Mathematical model of the cart inverted pendulum}

The cart inverted pendulum model is presented in Figure 1 [11].

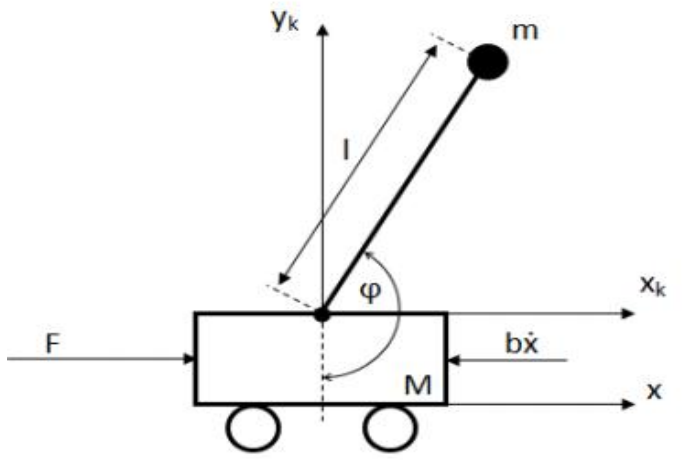

Figure 1. The cart inverted pendulum model

The equations of pendulum motion (a nonlinear system) can be formulated as:

$$
\begin{aligned}
& (M+m) \ddot{x}=-m l \varphi \ddot{x} \cos (\varphi)-m l \varphi^{2} \sin (\varphi)-b 1 \dot{x}+F \\
& \left(I+m l^{2}\right) \ddot{\varphi}=-m l \ddot{x} \cos (\varphi)-b 2 \dot{\varphi}-m g l \sin (\varphi) .
\end{aligned}
$$

Furthermore, we try to linearize the system differential equations. It is a linearization around the equilibrium point, in our case about vertical position $\varphi=\pi$. The prerequisite is that the system remains in the vicinity of this position. Let $\phi$ presents a deviation from the equilibrium point, we get $\phi=\pi+\theta$. When pendulthe um is in upright position $\cos \phi=\cos (\pi+\theta) \approx-1, \sin \phi=\sin (\pi+\theta) \approx-\theta, \dot{\phi}^{2}=\dot{\theta}^{2} \approx 0$.

Using above relation and from the nonlinear Equations (1) and (2) we obtained linearized equations of the proposed system as in (3) and (4).

$$
\begin{aligned}
& \left(I+m l^{2}\right) \ddot{\theta}+b 2 \dot{\theta}-m g l \theta=m l \ddot{x}, \\
& \ddot{x}(M+m)+b 1 \dot{x}-m l \ddot{\theta}=U .
\end{aligned}
$$


Note that $U$ has been substituted for the input force $F$. To obtain the transfer functions of the linearized system equations, we must first take the Laplace transform of the system equation assuming zero initial conditions. The resulting Laplace transforms are shown below:

$$
\begin{aligned}
& \left(I+m l^{2}\right) \Phi(s) s^{2}+b_{2} \Phi(s) s-m g l \Phi(s)=m l X(s) s^{2} \\
& (M+m) X(s) s^{2}+b_{1} X(s) s-m l \Phi(s) s^{2}=U(s)
\end{aligned}
$$

In this analysis, we set

$$
q=I(M+m)+M m l^{2}
$$

The transfer function of pendulum's position can determine from systems of Equations (5), (6) and (7) as the following:

$$
\frac{X(s)}{U(s)}=\frac{\frac{\left(I+m l^{2}\right)}{q} s^{2}+\frac{b_{2}}{q} s-\frac{m g l}{q}}{\left[s^{4}+\frac{(M+m) b 2+\left(I+m l^{2}\right) b_{1}}{q} s^{3}-\frac{\left[m g l(M+m)+b_{2} b_{1}\right]}{q} s^{2}-\frac{m g l b 1}{q} s\right]}
$$

Here transfer function of pendulum's angle can be formulated as:

$$
\frac{\Phi(s)}{U(s)}=\frac{\frac{m l}{q} s}{s^{3}+\frac{(M+m) b 2+\left(I+m l^{2}\right) b_{1}}{q} s^{2}-\frac{[m g l(M+m)+b 2 b 1]}{q} s-\frac{m g l b_{1}}{q}}
$$

We consider the equilibrium point $\varphi=0$, the pendulum become the normal form. The transfer functions of the pendulum's angle and position are presented as in the formula (10) and (11).

$$
\begin{aligned}
& \frac{\mathrm{X}(s)}{U(s)}=\frac{\frac{\left(I+m l^{2}\right)}{q} s^{2}+\frac{b_{2}}{q} s+\frac{m g l}{q}}{\left[s^{4}+\frac{(M+m) b 2+\left(I+m l^{2}\right) b_{1}}{q} s^{3}+\frac{\left[m g l(M+m)+b_{2} b_{1}\right]}{q} s^{2}+\frac{m g l b 1}{q} s\right]}, \\
& \frac{\Phi(s)}{U(s)}=\frac{-\frac{m l}{q} s}{s^{3}+\frac{(M+m) b 2+\left(I+m l^{2}\right) b_{1}}{q} s^{2}+\frac{[m g l(M+m)+b 2 b 1]}{q} s+\frac{m g l b_{1}}{q}}
\end{aligned} .
$$

For the transfer function above are valid these units, we assume that:

$$
P_{k}=\frac{\Phi(s)}{U(s)}\left[\frac{r a d}{N}\right], P_{v}(s)=\frac{X(s)}{U(s)}\left[\frac{m}{N}\right], \text { or } P_{k}=\frac{\Phi(s)}{U(s)}=\frac{180}{\pi}\left[\frac{\mathrm{deg}}{N}\right], P_{v}(s)=\frac{X(s)}{U(s)}\left[\frac{m}{N}\right]
$$

According to the transfer functions (8) and (9), the inverted pendulum with the equilibrium point $\varphi=\pi$ is unstable system. Due to unstable behavior of the upright equilibrium position, identification of the inverted pendulum represents a difficult task. However, the normal pendulum with the equilibrium point $\varphi=0$ is a stable system with respect to the transfer functions (10) and (11). Looking at the details, it is 
clearly seem that the absolute values of the coefficients of the transfer functions are the same for the pendulum's upright and downright positions. The difference is only in the sign of the coefficients. Based on this conclusion, the identification task of the nonlinear pendulum system can be applied on direct pendulum characteristics. In this paper, the response characteristics of the pendulum with the downright equilibrium point, will be consider as input data for the identification task in the next subsection [11]-[12].

\subsection{Real interpolation method (RIM)}

The real interpolation method is one of the methods, which works on mathematical descriptions of the imaginary area. The method is based on real integral transform as follow [13]:

$$
F(\delta)=\int_{0}^{\infty} f(t) e^{-\delta t} d t, \delta \in[C, \infty), C \geq 0
$$

which assigns to the original $f(t)$ the image $F(\delta)$ as a function of the real variable $\delta$. Formula of direct transform (12) can be considered as a special case of the direct Laplace transform by replacing the complex variable $p=\delta+j \omega$ for real $\delta$ variable. Another step towards the development of the instrumentation method is the transition from continuous functions $F(\delta)$ to their discrete form, using the computing resources and numerical methods. For these purposes, real interpolation method is represented by numerical characteristics $\left\{F\left(\delta_{i}\right)\right\}_{\eta}$. They are obtained as a set of values of the function $F(\delta)$ in the nodes $i=\overline{1, \eta}$, where $\eta$ is the number of elements numerical characteristics, called its dimension.

Selecting of interpolations $\delta_{i}$ is a primary step in the transition to a discrete form, which has a significant impact on the numerical computing and accuracy of problem solutions. Distribution of nodes in the simplest variant is uniform. Another important advantage of real interpolation method is cross-conversion properties [11]. It dues to the fact that the behavior of the function $F(\delta)$ for large values of the argument $\delta$ is determined mainly by the behavior of the original $f(t)$ for small values of the variable $t$. In the opposite case, the result is the same: the behavior of the function $F(\delta)$ for small values of the argument $\delta$ is determined mainly by the behavior of the original $f(t)$ for large values of the variable $t$ [13].

When considering the original $f(t)$ of dynamic characteristics of dynamic systems, formula (12) leads to an operator model, which under certain conditions can be considered as special cases of the models based on the Laplace transform. Thus, in (12) replacing of the function $f(t)$ by $k(t)$ - the impulse transient function of the dynamic system, we obtain its transfer function. From here we can find the elements of a discrete model of the system, and its transfer function by performing the discretization procedures for nodes $\delta_{i}, i \in \overline{1, \eta}$ :

$$
W\left(\delta_{i}\right)=\int_{0}^{\infty} k(t) e^{-\delta_{i} t} d t, i \in \overline{1, \eta}
$$

Function $W(\delta)$ is a real transfer function of control automatic systems, having an impulse transient response $k(t)$. Function $W(\delta)$ could be received based on determination of transfer function such as a relationship of the imaginary of output $Y(\delta)$ and $X(\delta)$ input signals

$$
W(\delta)=\frac{Y(\delta)}{X(\delta)}
$$

In which the imaginary of the output signal and the input signal is calculated from the original functions of the input $x(t)$ and output $y(t)$ signals:

$$
\begin{aligned}
& Y(\delta)=\int_{0}^{\infty} y(t) e^{-\delta t} d t, \\
& X(\delta)=\int_{0}^{\infty} x(t) e^{-\delta t} d t
\end{aligned}
$$

where $x(t)$ input signal, and $y(t)$ output signal of the system. 
The input-output relationship of the system has a form like below:

$$
Y(\delta)=W(\delta) X(\delta)
$$

\subsection{Identification of the inverted pendulum based on the RIM}

The inverted pendulum is an unstable system, investigation of this system based on input-output signal use two kinds of the input signal, namely impulse and steps responses. For unstable systems, identification of these systems using step response is not entirely suitable, because of the unstable transient response under the effect of the step response. Using impulse response can be used, however, in practice, the idea impulse response has challenging to implement. In this paper, the conversion impulse response is used for identification of nonlinear system. The inverted pendulum is used conversion impulse response having a form of transfer function:

$$
X(s)=M \frac{1-\exp (-\tau * s)}{s}
$$

where $M$ is magnitude, $\tau$ delay time.

Under the effect of a conversion impulse response (18) on the output of the system, response function is represented as a function $k(t)$. From formula (14) and (18), in the real form, the transfer function of the inverted pendulum has a form.

$$
W(\delta)=\frac{M \delta}{1-\exp (-\tau * \delta)} Y(\delta)
$$

where $Y(\delta)=\int_{0}^{\infty} k(t) e^{-\delta t} d t$ if response characteristics represents as a function $k(t)$, or $Y(\delta)=\sum_{i=1}^{N} k_{i}(t) e^{-\delta t_{i}} \Delta t$ $k_{i}(t)$ is a sample of $t_{i}$.

The linear transfer function of the angle of the inverted pendulum can be formulated as:

$$
\Phi_{L R}(s)=\frac{-B_{1} s}{s^{3}+A_{3} s^{2}+A_{2} s+A_{1}}
$$

The linear transfer function of the position of the inverted pendulum can be calculated as:

$$
X_{L R}(s)=\frac{B_{2} s^{2}+B_{1} s+B_{0}}{s^{4}+A_{3} s^{3}+A_{2} s^{2}+A_{1} s}
$$

The linear transfer function of angle and position of the inverted pendulum has a form of real interpolation transformation can be formulated as (22) and (23):

$$
\begin{gathered}
\Phi_{L R}(\delta)=\frac{-B_{1 \Phi} \delta}{\delta^{3}+A_{3} \delta^{2}+A_{2} \delta+A_{1}}, \\
X_{L R}(\delta)=\frac{B_{2 X} \delta^{2}+B_{1 X} \delta+B_{0 X}}{\delta^{4}+A_{3} \delta^{3}+A_{2} \delta^{2}+A_{1} \delta}
\end{gathered}
$$

Interestingly, the coefficients of the transfer function of the angle and position of the pendulum have the same number in the denominator. The identification process can be determined the coefficients of the denominator and separately the coefficients of the numerator. Firstly, identification of the transfer function of pendulum angle. Chosen structure and order of the linear identification like formula (20) and (21) or (22) and (23) in the real form. The number of the unknown coefficients for the angle of the pendulum is four; it means 
that the dimension of the elements numerical characteristics is four. In a simple case, the value of nodes given be calculated by nominal distribution in the range of $\left[\delta_{1}, \delta_{\eta_{\Phi}}\right]$, where $\eta_{\Phi}=4$.

$$
\delta_{i}=\delta_{1}+\frac{\delta_{\eta_{\Phi}}-\delta_{1}}{\eta_{\Phi}-1}(i-1), i=\overline{2, \eta_{\Phi}}
$$

Discretization of by real interpolation method in $\delta_{i}, i=\overline{1, \eta_{\Phi}}$.

$$
\frac{M \delta_{i}}{1-\exp \left(-\tau * \delta_{i}\right)} Y 1\left(\delta_{i}\right)=\frac{-B_{1 \Phi} \delta_{i}}{\delta_{i}^{3}+A_{3} \delta_{i}^{2}+A_{2} \delta_{i}+A_{1}}, i=\overline{1, \eta_{\Phi}},
$$

where $Y 1\left(\delta_{i}\right)=\int_{0}^{\infty} k_{\Phi}(t) e^{-\delta_{i} t} d t$, for the case, the transient characteristics is given a form of function with time. For the case, the transient characteristics is given a table or massive of values with sample time $\Delta t$.

$$
Y 1\left(\delta_{i}\right)=\sum_{i=1}^{N} k_{\Phi}\left(t_{i}\right) e^{-\delta_{i} t_{i}} \Delta t
$$

where, $\Delta t$-sampling time, $\mathrm{N}$-integrator. Here, we set replacement as:

$$
F 1\left(\delta_{i}\right)=\frac{M \delta_{i}}{1-\exp \left(-\tau * \delta_{i}\right)} Y 1\left(\delta_{i}\right), i=\overline{1, \eta_{\Phi}}
$$

From (14) and (16) we have a system of the equation like:

$$
F 1\left(\delta_{i}\right)=\frac{-B_{1 \Phi} \delta_{i}}{\delta_{i}^{3}+A_{3} \delta_{i}^{2}+A_{2} \delta_{i}+A_{1}}, i=\overline{1, \eta_{\Phi}}
$$

or

$$
\begin{aligned}
& F 1\left(\delta_{i}\right)\left(\delta_{i}^{3}+A_{3} \delta_{i}^{2}+A_{2} \delta_{i}+A_{1}\right)=-B_{1 \Phi} \delta_{i}, i=\overline{1, \eta_{\Phi}} \\
& \delta_{i}^{2} F 1\left(\delta_{i}\right) A_{3}+\delta_{i} F 1\left(\delta_{i}\right) A_{2}+A_{1}+\delta_{i} B_{1 \Phi}=-F 1\left(\delta_{i}\right) \delta_{i}^{3}, i=\overline{1, \eta_{\Phi}}
\end{aligned}
$$

Rewrite the system of equations of the unknown coefficients $A_{3}, A_{2}, A_{1}, B_{1 \Phi}$ Matrix M1:

$$
M 1=\left[\begin{array}{llll}
\delta_{1}^{2} F 1\left(\delta_{1}\right) & \delta_{1} F 1\left(\delta_{1}\right) & 1 & \delta_{1} \\
\delta_{2}^{2} F 1\left(\delta_{2}\right) & \delta_{2} F 1\left(\delta_{2}\right) & 1 & \delta_{2} \\
\delta_{3}^{2} F 1\left(\delta_{3}\right) & \delta_{3} F 1\left(\delta_{3}\right) & 1 & \delta_{3} \\
\delta_{4}^{2} F 1\left(\delta_{4}\right) & \delta_{4} F 1\left(\delta_{4}\right) & 1 & \delta_{4}
\end{array}\right]
$$

\section{Matrix N1}

$$
N 1=\left[\begin{array}{l}
-F 1\left(\delta_{1}\right) \delta_{1}^{3} \\
-F 1\left(\delta_{2}\right) \delta_{2}^{3} \\
-F 1\left(\delta_{3}\right) \delta_{3}^{3} \\
-F 1\left(\delta_{4}\right) \delta_{4}^{3}
\end{array}\right]
$$

Matrix X1 of the unknown coefficients 


$$
X 1=\left[\begin{array}{c}
A_{3} \\
A_{2} \\
A_{1} \\
B_{1 \Phi}
\end{array}\right]
$$

If the matrixes M1, N1 are known, the matrix X1 can be determined as the following:

$$
X 1=M 1^{-1} N 1
$$

The identification task leads to solving a system of equation of $\eta$ unknown coefficients of the ransfer function. Identification of transfer function of pendulum's position. We already determined the coefficient of denominator $\mathrm{n}$ above step, in terms of simplicity, requirement determines unknown coefficients of the numerator of the transfer function of pendulum's position. The number of characteristics is $\eta_{X}=3$. Transfer function of pendulum's position has the form as (23):

$$
X_{L R}(\delta)=\frac{B_{2 X} \delta^{2}+B_{1 X} \delta+B_{0 X}}{\delta^{4}+A_{3} \delta^{3}+A_{2} \delta^{2}+A_{1} \delta}
$$

Obviously, in order to reduce identification task, the coefficient $A_{1}, A_{2}$, and $A_{3}$ has already determined in the first section, the identification task remains only determine coefficients of the numerator of the transfer function of pendulum's position $B_{0}, B_{1}$, and $B_{2}$. The identification procedure of transfer function of pendulum's position illustrates following steps. If response characteristics are given a massive of time, with the sample time $\Delta t, k_{X}\left(t_{i}\right)$ value of pendulum position at $t_{i}$ and $\mathrm{N}$-number of samples, numerical characteristics are calculated by following form:

$$
Y 2\left(\delta_{i}\right)=\sum_{i=1}^{N} k_{X}\left(t_{i}\right) e^{-\delta_{i} t_{i}} \Delta t
$$

\section{RESULTS AND DISCUSSION}

The inverted pendulum has parameter as in Table 1 .

Table 1. Parameters of the Pendulum System

\begin{tabular}{lcc}
\hline \multicolumn{1}{c}{ Parameter } & Value & Unit \\
\hline Mass of the cart $(\mathrm{M})$ & 0.5 & $\mathrm{Kg}$ \\
Mass of the pendulum (m) & 0.2 & $\mathrm{Kg}$ \\
Length of the pendulum (l) & 0.3 & $\mathrm{M}$ \\
Coefficient of friction for the cart (b1) & 0.2 & $\mathrm{Nm}-1 \mathrm{~s}-1$ \\
Coefficient of friction for the pendulum (b2) & 0.002 & $\mathrm{Nrad}-1 \mathrm{~s}-1$ \\
Mass moment of inertia of the pendulum (I) & 0.006 & $\mathrm{~kg} / \mathrm{m} 2$ \\
Gravitation force (g) & 9.81 & $\mathrm{~m} / \mathrm{s} 2$ \\
Force applied to the cart $(\mathrm{F})$ & - & $\mathrm{N}$ \\
Position of the cart $(\mathrm{x})$ & - & $\mathrm{m}$ \\
Pendulum angle $(\varphi)$ & - & $\mathrm{deg}$. \\
\hline
\end{tabular}

In this example we will compare results of the nonlinear modeling, describing as the formulas (1) and (2), with a linear model, solving by the analytical approximation as formula (10) and (11) and with a RIM model, which is identified by RIM. The pendulum has a set of parameters as the Table 1. On input of the models, the rectangular input acceleration signal has $1 \mathrm{~N}$ in time $1 \mathrm{~s}$ in the Figure 2(a).

In the Figure 3, obviously, the RIM model and analytical linear model highly fit with nonlinear model in the beginning of the transient process. However, in the end of the transient process, the linear model represents higher in fitness comparing with the RIM model. Moreover, Figure 4 shows that the RIM model has more accuracy at the beginning of the span time [0-7 s]. However, the fitness of RIM model is reducing with the increase of time in the range 7-70 s. From the results, the maximum error of linear is 0.24 approximation by RIM is 0.45 . Generally, the linear model represents a higher accuracy model than RIM 
model when the angle deviation from the downright equilibrium position is lower than $15^{\circ}$. The position of the pendulum versus time is presented in Figure 5. Interestingly, the RIM model of position of pendulum illustrates higher fitness level of nonlinear model than the analytical linear model. According to the absolute errors of the analytical linear model and the RIM model presented in the Figure 6. The error of the RIM model is considerably lower than of the analytical linear model in the time range [0-10 s]. In the rest of the considered settling time, the analytical model is around 1.5 times as high as the RIM model. The maximum error of the analytical and RIM models account for 0.038 and 0.026 , respectively

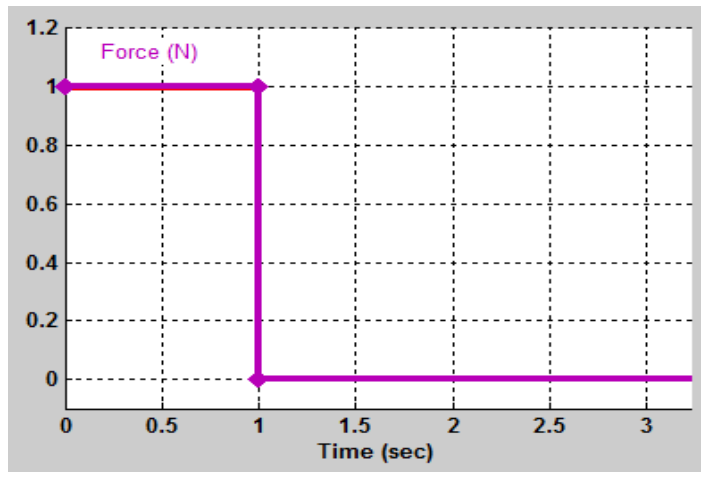

(a)

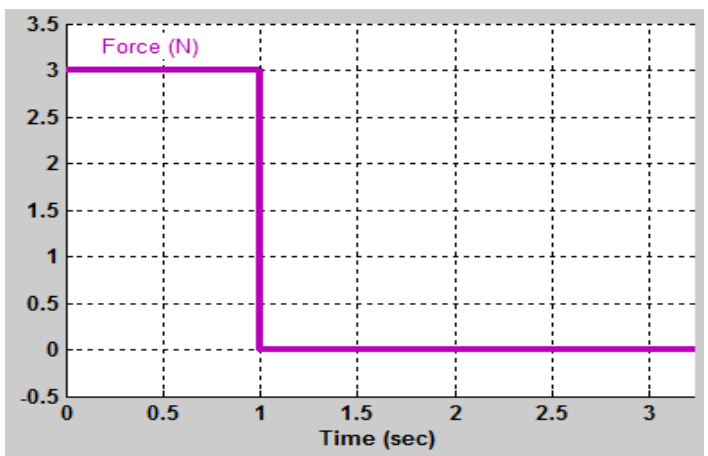

(b)

Figure 2. The input acceleration signal

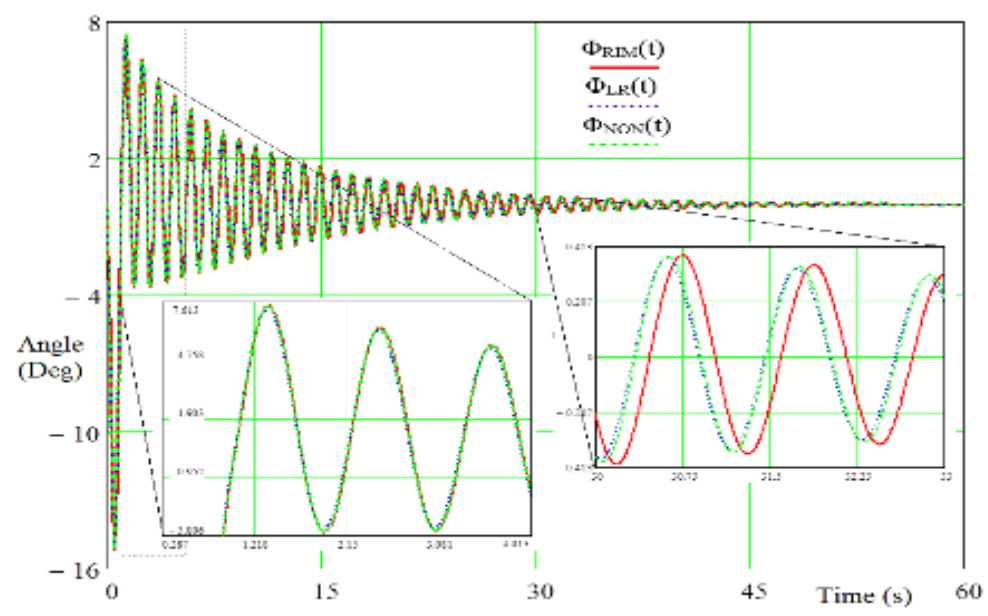

Figure 3. Response characteristics of nonlinear - green-dot line, analytic - blue-dot-line, and identification by RIM -red line

The input acceleration force with $3 \mathrm{~N}$, illustrated in Figure 2(b) is active on a cart during 1 second, based on the samplings of the nonlinear model, we find the approximation model by RIM for the new set of data. For identification of the deviation angle of pendulum, the nodes considered in the range $\delta_{i}=[1,2.33,3.67,5]$, sample time $\Delta t=0.001 \mathrm{~s}$ and settling time $70 \mathrm{~s}$. Then making a comparison with the original nonlinear model, linear model, and RIM model, we show the results as Figure 7 and Figure 8. Overall, the Figure 7 show that the RIM model represents more accuracy than the analytical linear model, especially in the range $0-10$ s of the settling time. Interestingly, with a high displacement of the angle from the equilibrium point $\varphi=0$, the RIM model represents more accuracy then linear model. Maximum error of the linear model and the RIM model is 9.17 and 8.62, respectively. 


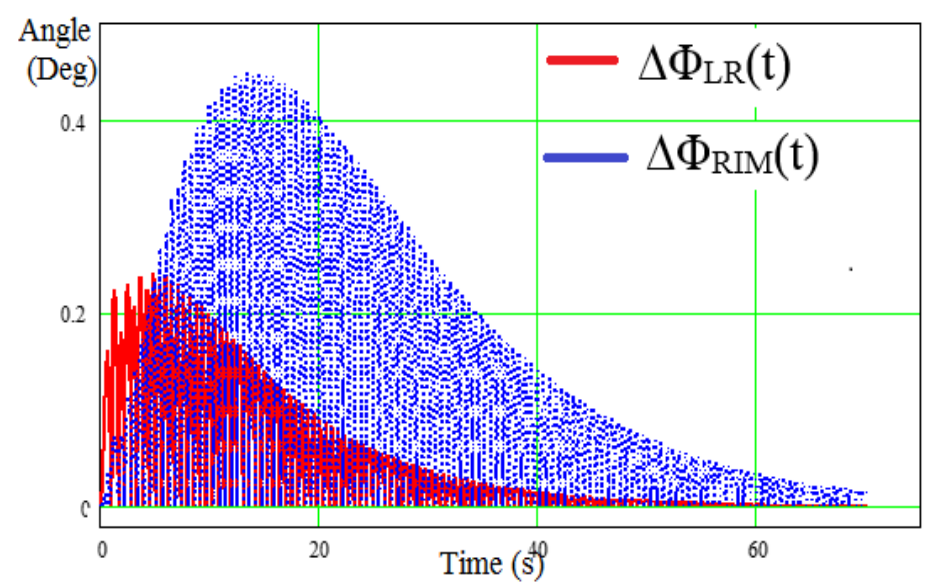

Figure 4. Approximation error: Linearization error-red line, approximation error by RIM - blue line

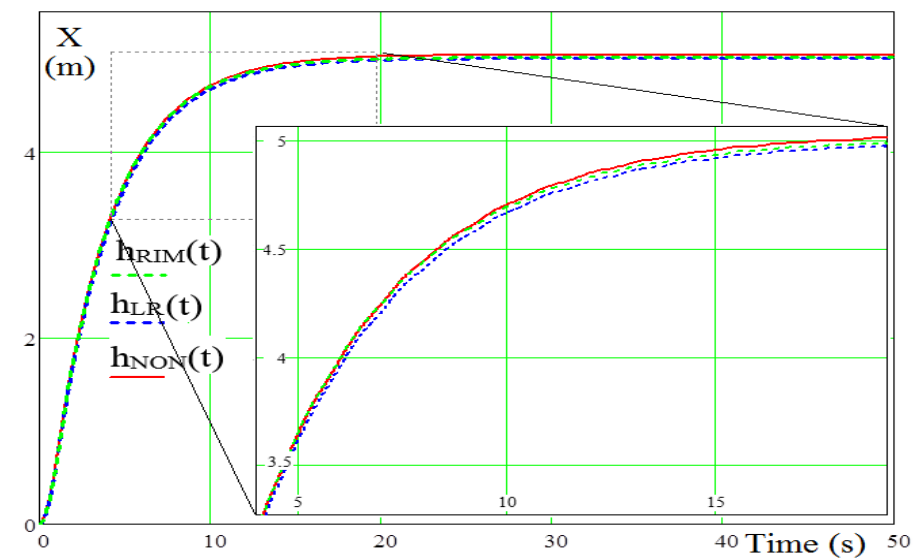

Figure 5. The position of the pendulum: Nonlinear model red-line, linearization model- blue line, approximation model by RIM - green line

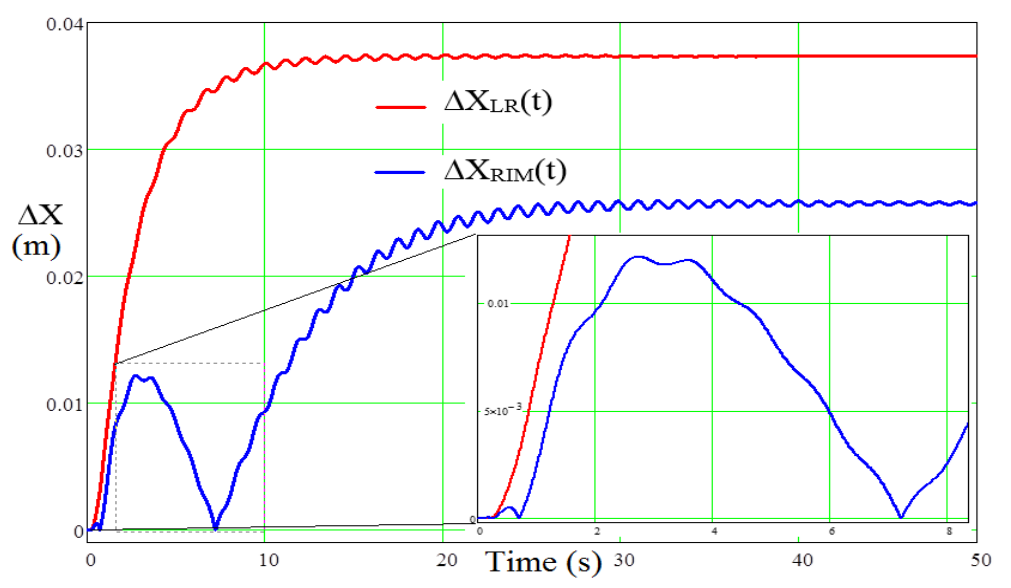

Figure 6. The error of models: linearization model - red line, approximation model by RIM - blue line 


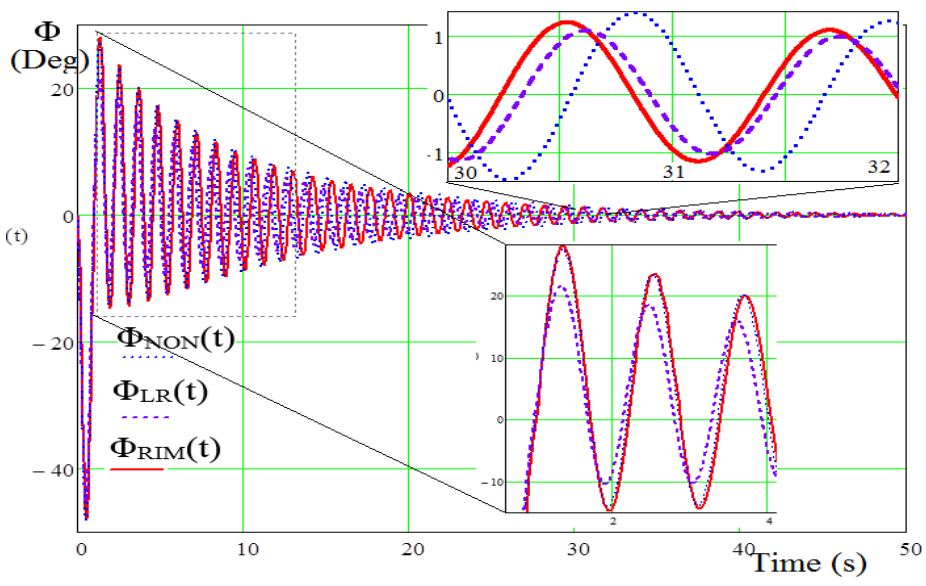

Figure 7. The angle of the pendulum: nonlinear model - blue dot line, linear model - violet dot line, approximation by RIM - red line

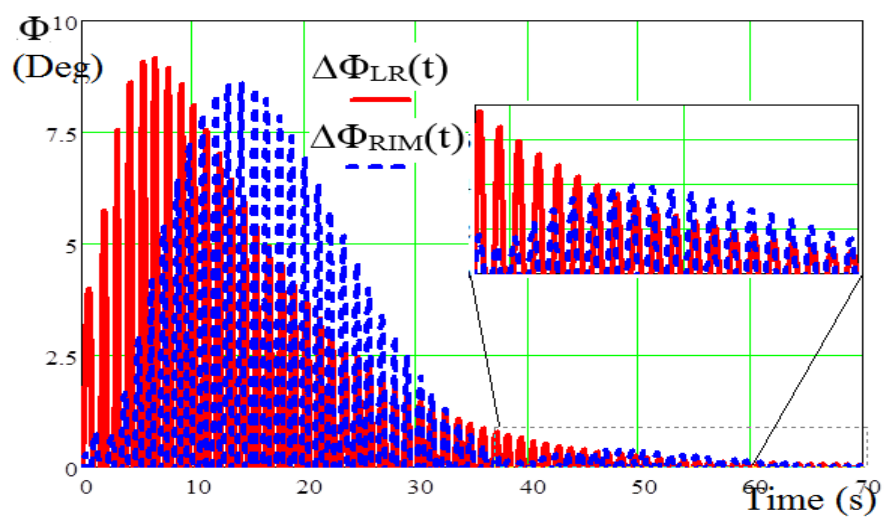

Figure 8 . The error of angle - linear model - red line, RIM model - blue line

Position of the pendulum under acting by $3 \mathrm{~N}$ during 1 second presents in Figure 9. Obviously, the RIM model has a higher fitness than the analytical linear model in the beginning of the settling time [0-10 s]. In the end of the settling time the fitness of both models is approximately the same. Looking at more details in the Figure 10, the fitness of the RIM model is higher than of the analytical model in time range [0-20 s].

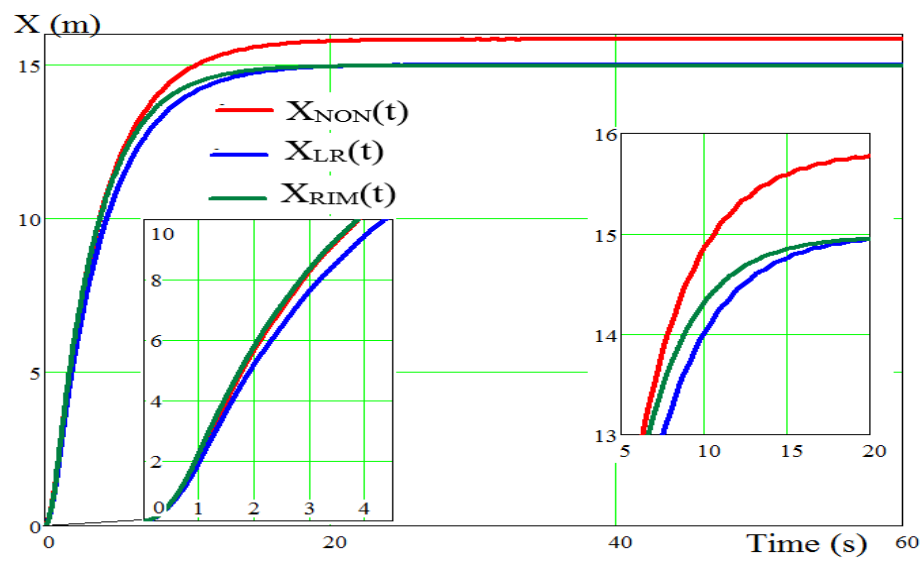

Figure 9. The position of the pendulum: nonlinear model -red line, linear model - blue line, RIM model green line 


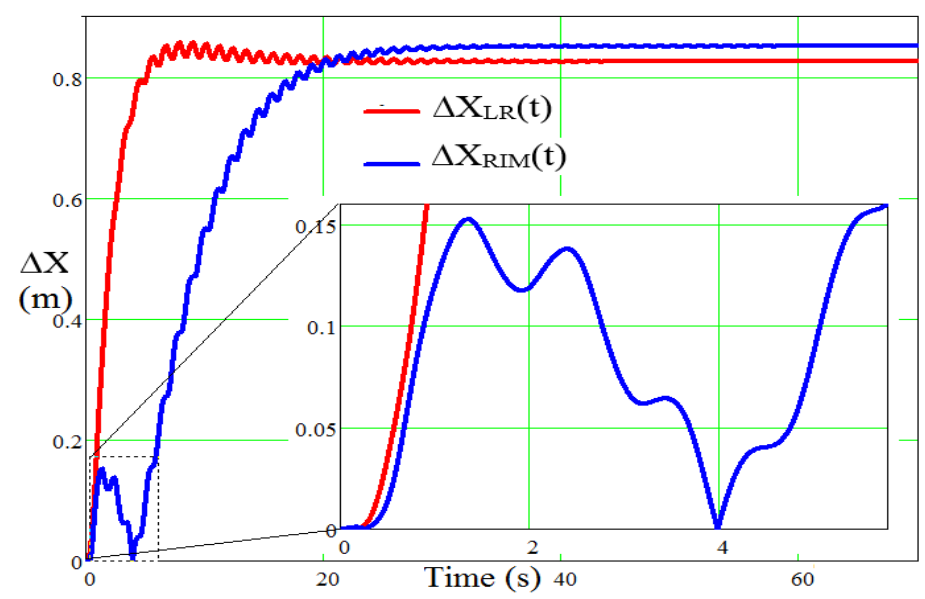

Figure 10. The error of model- linearization model -red line; approximation model by RIM - blue line

However, in the further transient time, the RIM model is slightly less accuracy than the analytical linear model. The maximum error of the deviation of the pendulum's position of both models is the same at about 0.83 . According to the two careful samples, the models identified by RIM represent higher accuracy than model of analytical linear approach in cases of that the deviation of pendulum accounts for higher $45^{\circ}$. RIM provides adaptively tool to obtain the linear transfer function of non-linear system based on transient characteristics, which has significant advantage compare analytical methods. The method which is based on expression from physical laws of dynamic pendulum. Another advantage of RIM is that the method is conducted in real domain, which is more effective compared to other methods implement in imaginary and frequency domains.

\section{CONCLUSION}

In this paper, the inverted pendulum system by using real interpolation method (RIM) algorithm is investigated. After the identification of the inverted pendulum system by using the real interpolation method (RIM) algorithm is analyzed, the analytical algorithm derived by Simulink Mat Lab Software. From the results, it is found that the inverted pendulum system by using real interpolation method (RIM) algorithm is simplicity, low computer source requirement, high accuracy. It can be found like the prospective solution for the inverted pendulum research direction in the near future.

\section{REFERENCES}

[1] Glück, Tobias, Andreas Eder, and Andreas Kugi, "Swing-up Control of a Triple Pendulum on a Cart with Experimental Validation," Automatica 49, no. 3 (03 2013): 801-08. doi:10.1016/j.automatica.2012.12.006.

[2] Aracil, J., J.á. Acosta, and F. Gordillo, “A Nonlinear Hybrid Controller for Swinging-up and Stabilizing the Furuta Pendulum," Control Engineering Practice 21, no. 8 (08 2013): 989-93. doi:10.1016/j.conengprac.2013.04.001.

[3] Åström, K.j., and K. Furuta, "Swinging up a Pendulum by Energy Control," Automatica 36, no. 2 (02 2000): 28795. doi:10.1016/s0005-1098(99)00140-5.

[4] Shehu, Muhammad, Mohd Ridzuan Ahmad, Auwal Shehu, and Ahmad Alhassan, "LQR, Double-PID and Pole Placement Stabilization and Tracking Control of Single Link Inverted Pendulum," 2015 IEEE International Conference on Control System, Computing and Engineering (ICCSCE), 112015. doi:10.1109/iccsce.2015.7482187.

[5] S., Gopikrishnan, Ameya Anil Kesarkar, and N. Selvaganesan, "Design of Fractional Controller for Cart-pendulum SIMO System," 2012 IEEE International Conference on Advanced Communication Control and Computing Technologies (ICACCCT), 08 2012. doi:10.1109/icaccct.2012.6320764.

[6] Mishra, Sunil Kumar, and Dinesh Chandra, "Stabilization and Tracking Control of Inverted Pendulum Using Fractional Order PID Controllers," Journal of Engineering 2014 (2014): 1-9. doi:10.1155/2014/752918.

[7] Prasad, Lal Bahadur, Barjeev Tyagi, and Hari Om Gupta, "Optimal Control of Nonlinear Inverted Pendulum Dynamical System with Disturbance Input Using PID Controller \&amp; LQR,” 2011 IEEE International Conference on Control System, Computing and Engineering, 11 2011. doi:10.1109/iccsce.2011.6190585.

[8] Kharola, Ashwani, Pravin Patil, Suyashi Raiwani, and Deepak Rajput, "A Comparison Study for Control and Stabilisation of Inverted Pendulum on Inclined Surface (IPIS) Using PID and Fuzzy Controllers," Perspectives in Science 8 (09 2016): 187-90. doi:10.1016/j.pisc.2016.03.016. 
[9] Tatikonda, Ravi Chandra, Venkata Praveen Battula, and Vijay Kumar, "Control of Inverted Pendulum Using Adaptive Neuro Fuzzy Inference Structure (ANFIS)," Proceedings of 2010 IEEE International Symposium on Circuits and Systems, 05 2010. doi:10.1109/iscas.2010.5537234.

[10] Elsayed, Belal A., Mohsen A. Hassan, and Saad Mekhilef, "Fuzzy Swinging-up with Sliding Mode Control for Third Order Cart-inverted Pendulum System," International Journal of Control, Automation and Systems 13, no. 1 (12, 2014): 238-48. doi:10.1007/s12555-014-0033-4.

[11] Strakos, Premysl, and Jiri Tuma, "Mathematical Modelling and Controller Design of Inverted Pendulum," 2017 18th International Carpathian Control Conference (ICCC), 05 2017. doi:10.1109/carpathiancc.2017.7970431.

[12] Peker, Fuat, and Ibrahim Kaya, "Identification and Real Time Control of an Inverted Pendulum Using PI-PD Controller," 2017 21st International Conference on System Theory, Control and Computing (ICSTCC), 102017. doi:10.1109/icstcc.2017.8107130.

[13] Goncharov, Nguyen Quang Dung, Than Viet Dung, Identification of object with distributed parameters, Theory and practice in the physical, mathematical and technical sciences-XXIV International Scientific and Practice Conference, London, May, 2012. doi:10.18007/gisap:tsca.v0i8.1421.

[14] Tin, Phu Tran, Tran Hoang Quang Minh, Tan N. Nguyen, and Miroslav Voznak, "System Performance Analysis of Half-Duplex Relay Network over Rician Fading Channel," TELKOMNIKA (Telecommunication, Computing, Electronics and Control). 16, no. 1 (02, 2018): 189. doi:10.12928/telkomnika.v16i1.7491.

[15] Rashid, Tarique, Sunil Kumar, Akshay Verma, Prateek Raj Gautam, and Arvind Kumar, "Pm-EEMRP: Postural Movement Based Energy Efficient Multi-hop Routing Protocol for Intra Wireless Body Sensor Network (IntraWBSN)," TELKOMNIKA (Telecommunication, Computing, Electronics and Control) 16, no. 1 (02, 2018): 166. doi:10.12928/telkomnika.v16i1.7318.

[16] A. F. Morabito, "Power Synthesis of Mask-Constrained Shaped Beams Through Maximally-Sparse Planar Arrays," TELKOMNIKA (Telecommunication, Computing, Electronics and Control), vol. 14, n. 4, pp. 1217-1219, 2016. 